\title{
UMA CARACTERIZAÇÃO DE GRAFOS IMERSÍVEIS
}

\author{
Walter Julio Cortez Morales \\ Depto. de Ciências de Computação e Estatística / IBILCE \\ Universidade Estadual Paulista (UNESP) \\ São José do Rio Preto - SP \\ walter@ibilce.unesp.br
}

Recebido em 02/2002; aceito em 12/2004 após 4 revisões

Received February 2002; accepted December 2004 after 4 revisions

\begin{abstract}
Resumo
Este trabalho é motivado pelo resultado de Berge, que é uma generalização do teorema de Tutte o qual expressamos na forma: Dado o grafo $G$ de ordem $|\mathrm{V}(\mathrm{G})|$ e $v(\mathrm{G})$ o número de arestas em um emparelhamento máximo, existe um conjunto $X$ de vértices de $G$ tal que $|V(G)|+|X|-\omega(G X X)-$ $2 v(\mathrm{G})=0$, onde $\omega(G \backslash X)$ é o número de componentes de ordem ímpar de $G \backslash X$. Tal expressão chamamos a equação de Tutte-Berge associada de $G$, e escrevemos simplesmente $T(G ; X)=0$. Os grafos podem ser classificados a partir das soluções da equação de Tutte-Berge. Um grafo $G$ é chamado imersivel se, e somente se, $T(G ; X)=0$ possui pelo menos um conjunto solução não vazio de vértices, e $G$ é denominado não imersível se, e somente se, o conjunto vazio é a única solução de $T(G ; X)=0$. O resultado principal deste artigo é a caracterização de grafos imersiveis pelos conjuntos antifatores completos, além disso, provamos que os grafos fatoráveis estão contidos na classe dos imersíveis.
\end{abstract}

Palavras-chave: grafos; imersível; antifator completo; fatorável; equação de Tutte-Berge.

\begin{abstract}
This paper is motivated by the result of Berge who generalized Tutte's theorem which states that: Given a graph $G$ with $|V(G)|$ vertices and $v(\mathrm{G})$ the number of edges in a maximum matching, then there is a subset $X \subseteq V(G)$ such that $|V(\mathrm{G})|+|X|-\omega(G \backslash X)-2 v(G)=0$, where $\omega(G \backslash X)$ denotes the number of odd components of $G \backslash X$, such expression is called Tutte-Berge's equation associated to $G$, denoted by $T(G ; X)=0$. These graphs are then studied from solutions of $T(G ; X)=0$. A graph $G$ is called immersible graph if and only if, its associated equation $T(G ; X)=0$ has at least one non-emptyset for $X$, and it is non-immersible graph if and only if, the unique solution to $T(G ; X)=0$ is the emptyset. The main result of this work is the characterization of immersible graphs via complete antifactor sets, moreover we prove that factorizable graphs are included in the class of immersible graphs.
\end{abstract}

Keywords: graphs; immersible; complete antifactor; factorizable; Tutte-Berge's equation. 


\section{Introdução}

O presente trabalho foi motivado pelo resultado de Berge [Ber57], que é uma generalização do teorema de Tutte [Tut47] o qual expressamos na forma: Dado o grafo $G$ de ordem $|V(G)|$ e $v(G)$ o número de arestas em um emparelhamento máximo, então existe um conjunto $X$ de vértices de $G$ tal que $|V(G)|+|X|-\omega(G \backslash X)-2 \mathrm{v}(G)=0$, onde $\omega(G \backslash X)$ é o número de componentes de ordem ímpar de $G \backslash X$. Tal expressão chamamos a equação de Tutte-Berge associada de $G$ e escrevemos simplesmente $T(G ; X)=0$. Classificamos os grafos a partir do conjunto soluções da equação associada. Um grafo $G$ é chamado imersivel se, e somente se, a equação $T(G ; X)=0$ associada de $G$ possui pelo menos um conjunto solução não vazio de vértices, e $G$ é denominado não imersivel se, e somente se, Ø é a única solução de $T(G ; X)=0$ (denotaremos com o símbolo Ø o conjunto vazio). Duas arestas são disjuntas em $G$, se elas não compartilham nenhum vértice em comum, e um subconjunto $M \subseteq E(G)$ é chamado um emparelhamento se as arestas de $M$ são mutuamente disjuntas. Um emparelhamento $M$ em $G$ é máximo se não existe outro emparelhamento $M^{\prime}$ tal que $\left|M^{\prime}\right|>|M|(|A|$ denota a cardinalidade de um conjunto $A$ ). A cardinalidade de um emparelhamento máximo em $G$, denotado por $v(G)$ (ou simplesmente por $v$ se não precisar especificar o grafo $G$ ) é o número emparelhamento de $G$. Um subconjunto $S \subseteq V(G)$ é denominado antifator de $G$ se $\omega$ $(G \backslash S)>|\mathrm{S}|$. Além disso, se o conjunto $S$ verifica a igualdade $\omega(G \mid S)=|S|$, então $S$ é chamado um antifator completo de $G$.

Neste artigo, descrevemos uma caracterização de grafos imersiveis pelos conjuntos antifatores completos, e provamos que a classe de grafos fatoráveis, definida adiante, forma uma subclasse destes grafos imersiveis. Todos os grafos considerados são finitos, conexos, simples e não direcionados. A seguir serão dados alguns resultados e definições necessários para o bom entendimento deste trabalho. Outras definições usadas no texto mas não mencionadas nesta seção podem ser encontradas em Boaventura Netto [Boa03], Bondy [Bon95], Cornuéjols [Cor88] e Lovász \& Plummer [Lov86].

Um grafo não orientado ou simplesmente um grafo $G$ é um par ordenado $(V(G), E(G))$, onde $V(G)$ é um conjunto não vazio de elementos chamados vértices e $E(G)$ é o conjunto de arestas formado por pares não ordenados de vértices. As cardinalidades $|V(G)| \mathrm{e}|E(G)|$ são denominados ordem e o tamanho, respectivamente, de $G$. Para qualquer $x \in V(G)$, seja $E_{G}(x)=\{x y ; x y \in E(G)\}$. O grau de $x$ em $G$ será denotado por $d_{G}(x)$ e é igual a $\left|E_{G}(x)\right|$.

Denotaremos por $K_{n}, C_{n}$ e $K_{n, m}$, respectivamente, grafo completo, ciclo sobre $n$ vértices e o grafo bipartido completo com partes de tamanhos $n$ e $m$. Em particular, $K_{l, n}$ é uma $n$-estrela, cujo centro é o vértice de grau $n$.

Uma componente conexa $F$ de um grafo $G$ é chamado ímpar (par) se $|V(F)|$ é ímpar (par).

A coleção de todas as componentes conexas é denotada por $C=C_{0} \cup C_{1}$ (ou $C(G)$ se for preciso especificar o grafo $G$ ), onde $C_{1}$ e $C_{\mathrm{o}}$ são, respectivamente, as coleções de componentes conexas de ordem ímpar e par, e notamos $\omega(G)=\left|C_{l}\right|$ o número de componentes de ordem ímpar. Para um subconjunto $X \subseteq V(G)$, denota $G[X]$ o subgrafo induzido de $G$ por $X$ e $G \backslash X=G[V(G) \backslash X]$ é o grafo obtido de $G$ pela remoção de todos os vértices de $X$ e conjuntamente as arestas que incidem em $X$. Um t-emparelhamento é um emparelhamento $M=\left\{x_{1} y_{1}, \ldots, x_{t} y_{t}\right\}$ com $t$ arestas. Um emparelhamento $M$ em $G$ é perfeito se $|V(G)|=2|M| \mathrm{e}$ dizemos que o grafo $G$ é fatorável, se $G$ contém emparelhamento perfeito. 
Um dos resultados mais importantes sobre os grafos fatoráveis é sem dúvida o seguinte teorema devido a Tutte [Tut47].

Teorema 1.1. Um grafo $G=(V(G), E(G))$ possui um emparelhamento perfeito se, e somente se,

$$
\omega(G \backslash X) \leq|X|, \quad \forall X \subseteq V(G) .
$$

A generalização deste teorema foi dada por Berge [Ber57], conforme o seguinte teorema.

Teorema 1.2. Dado o grafo $G=(V(G), E(G))$, então $2 v(G)=\min _{X \subseteq V(G)}\{|V(G)|+|X|-$ $\omega(G \backslash X)\}$.

No que se segue, um vértice $v$ de um grafo $G$ (no qual se considera um emparelhamento $M$ ) é dito $M$-saturado se alguma aresta de $M$ é incidente com $v$. Caso contrário, $v$ é chamado M-não-saturado.

A deficiencia de um emparelhamento $M$ em $G$ é o número de vértices no-saturados por $M$, e a deficiência de $G, \operatorname{def}(G)$ é definida como $\operatorname{def}(G)=|V(G)|-2 v(G)$. O seguinte corolário (que é equivalente ao teorema 1.2) é conhecido como a fórmula de Berge.

Corolário 1.1. Consideremos o grafo $G=(V(G), E(G))$, então $|V(G)|-2 v(G)=\max _{\mathrm{X} \subseteq \mathrm{V}(\mathrm{G})}$ $\{\omega(G \mid X)-|X|\}$.

$\mathrm{O}$ artigo está organizado como segue. Na seção 2, introduzimos definições de $n$-coleções, grafos imersíveis e da equação de Tutte-Berge e provamos dois resultados conhecidos usando a nossa terminologia, que serão usados no presente trabalho, [Bru87] e [Die97, pp.41]. Na seção 3, apresentamos a prova de nosso resultado principal que mostra a caracterização dos grafos imersiveis, mas antes definimos o conjunto antifator completo de um grafo e provamos a seguinte afirmação: todo conjunto não vazio de vértices de um grafo $G$, que verifica a condição estrita de Tutte, não é uma solução da equação de Tutte-Berge associada de $G$. Na Seção 4 será mostrado que os grafos imersiveis contêm a classe dos fatoráveis.

\section{Equação de Tutte-Berge e Grafos Imersíveis}

Em Cortez-Morales [Cor99], para um inteiro dado $n \geq 3$ definimos uma $n$-coleção como sendo uma família de grafos conexos e simples, e denotada por $\mathrm{H}^{\mathrm{n}}(m, v(G))$, com os parâmetros inteiros: $m$ é o número de arestas e $v(G)$ é o tamanho do maior emparelhamento do grafo, onde,

$$
n-1 \leq m \leq \frac{n(n-1)}{2} \quad \text { e } \quad 1 \leq v(G) \leq\left\lfloor\frac{n}{2}\right\rfloor
$$

respectivamente, $(\lfloor x\rfloor$ denota o maior inteiro menor ou igual a $x)$.

A fim de clarear a notação, embora seja um abuso, escrevemos sob a estrutura tipo polinomial nos parâmetros $m$ e $v(G)$,

$$
H^{n}(m, v(G))=\sum_{m=n-1, n, \ldots, \frac{n(n-1)}{2}} \sum_{1 \leq v(G) \leq \frac{n}{2}} \Gamma_{m, v(G)}
$$


onde, $\Gamma_{m, v(G)}$ é uma família de grafos conexos mutuamente não isomorfos com o mesmo número de vértices $n$ (fixado) e todos eles tendo o mesmo tamanho $m$ e o mesmo número emparelhamento $v(G)$.

Para $n=5$, onde $4 \leq m \leq 10$ e $1 \leq v(G) \leq 2$ temos $H^{5}(m, v(G))=\Gamma_{4,1}+\Gamma_{4,2}+\Gamma_{5,2}+\Gamma_{6,2}+$ $\Gamma_{7,2}+\Gamma_{8,2}+\Gamma_{9,2}+\Gamma_{10,2}$

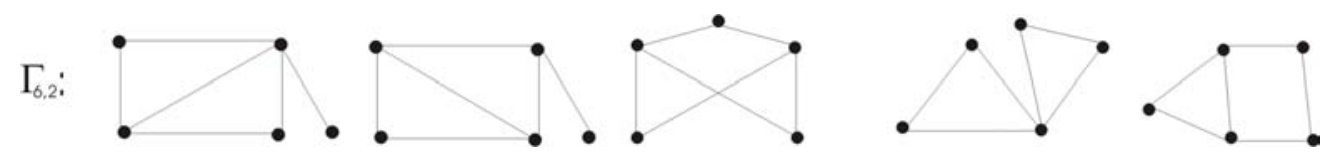

Figura 1 - Na 5-coleção $\mathrm{H}^{5}(m, v(G))$ tomamos a família $\Gamma_{6,2}$ com $m=6$ e $v(G)=2$ de grafos conexos e mutuamente não isomorfos.

Nos referiremos a $G$ como sendo um grafo conexo e simples de ordem $n$, ou de maneira equivalente podemos dizer que $G$ é um membro de $\mathrm{H}^{n}(m, v(G))$ ou $G \in \mathrm{H}^{n}(m, v(G))$.

Definição 2.1. Para $G \in \mathrm{H}^{n}(m, v(G))$ e $X \subseteq V(G)$, a expressão $T(G ; X)=|V(G)|+|X|-$ $\omega(G \backslash X)-2 v(G)=0$, é chamada equação de Tutte-Berge associada de $G$. Um subconjunto $S$ de vértices de $V(G)$ é uma solução para $T(G ; X)=0$, se a igualdade é verificada quando $X=S$. O grafo $G$ é chamado imersível se, e somente se, sua equação associada $T(G ; X)=0$ possui pelo menos um conjunto solução não vazio de vértices, e $G$ é denominado não imersível se, e somente se, $T(G ; X)=0$ admite como a única solução o conjunto vazio.

Denotando-se por $G_{\mathrm{im}}$ e $G_{n-i m}$ as classes de grafos imersíveis e não imersíveis, respectivamente, podemos escrever $H^{n}(m, v(G))=G_{i m}+G_{n-i m}$, se ambas classes de grafos não forem vazios.

O nome imersível refere-se ao ato de buscar alguma coisa imersa, por exemplo, entre os conjuntos de vértices não vazios de um grafo. Além disso, há uma certa analogia com a noção de dependência linear de álgebra linear.

Por exemplo: Na Figura 1, os três primeiros desenhos representam grafos imersíveis e os outros dois últimos grafos não imersíveis. Na Figura 2, o grafo completo $K_{3}$ é não imersível, enquanto para qualquer aresta e de $K_{3}$, o grafo $K_{3}$-e é imersível.
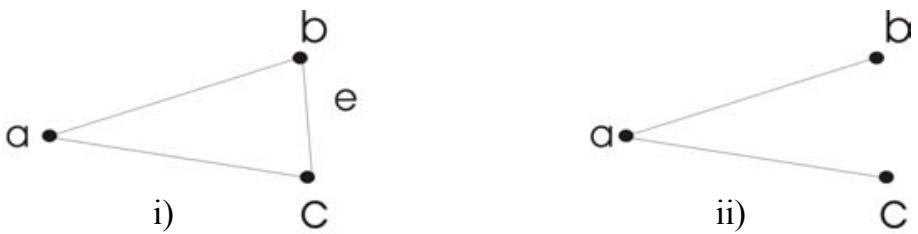

Figura 2 - i) $\mathrm{O}$ grafo completo $\mathrm{K}_{3}$ não é imersivel, pois $\mathrm{T}\left(\mathrm{K}_{3} ; \mathrm{X}\right)=0$ tem como a única solução $\varnothing$, ii) $\mathrm{O}$ grafo $\mathrm{K}_{3}$-e é imersivel, pois, além da solução $\varnothing$, o conjunto $X=\{\mathrm{a}\}$ é outra solução da equação $T\left(\mathrm{~K}_{3-} e ; X\right)=0$.

A proposição seguinte prova que $|V(G)|+|X|-\omega(G \backslash X)$ é um inteiro par. 
Proposição 2.1. [Bru87, p.57] Seja $G=(V(G), E(G))$ um grafo conexo, então $|V(G)|+|X|-\omega$ $(G \backslash X)$ é um número inteiro par, para todo subconjunto $X \subseteq V(G)$.

Demonstração. Vamos argumentar por contradição. $|V(G)|-[\omega(G \backslash S)-|S|]$ é um inteiro ímpar se, e somente se, acontecem um dos dois casos:

(a) $|V(G)|$ é ímpar e $[\omega(G \backslash S)-|S|]$ é par.

Neste caso, temos dois subcasos

i. $\omega(G \backslash S)$ e $|S|$ são ambos pares.

Nós lembramos que definimos $\mathrm{C}_{\mathrm{o}}$ e $\mathrm{C}_{1}$, respectivamente, como as coleções de componentes conexas de ordens ímpares e pares.

Dado que $\omega(G \backslash S)=2 t,|S|=2 r$ e $\mathrm{C}^{1}, \mathrm{C}^{2}, \mathrm{C}^{3}, \ldots, \mathrm{C}^{2 t}$ são as $2 t$ componentes conexas ímpares de $G \backslash S$. Assim, o valor

$$
|V(G)|=|S|+\sum_{i=1}^{2 t}\left|C^{i}\right|+\sum_{C \in C_{o}}|C|
$$

é um número par, porque $|S|$ é par, a soma de um número par de inteiros ímpares é par e cada componente conexa de $\mathrm{C}_{\mathrm{o}}$ possui tamanho par.

ii. $\omega(G \backslash S)$ e $|S|$ são ambos ímpares.

Analogamente este caso também conduz a uma contradição.

(b) $|V(G)|$ é par e $[\omega(G \backslash S)-|S|]$ é ímpar.

Para provar esta parte da contradição é suficiente mostrar que o fato de $|V(G)|$ ser par, implica em $\omega(G \backslash S)$ e $|S|$ possuírem a mesma paridade. Com efeito, fixada $p$ uma paridade para $\omega(G \backslash S)$, temos como conseqüência que, o número total de vértices na coleção de componentes ímpares de $G \backslash S$ possui paridade $p$. Em adição ao número total de vértices na coleção de componentes pares ser par, concluímos que $\omega(G \backslash S)$ possui paridade $p$. Como $|V(G)|$ é par temos que $|S|$ possui paridade $p$. O que encerra o argumento de contradição.

Observação 2.1. [Bru87, p.57, prop.4.1.8] Se $S$ é uma solução para $T(G ; X)=0$, isto não implica que $G \backslash S$ não tenha componentes pares. Porém, se $S$ for maximal, então $G \backslash S$ tem somente componentes ímpares.

Demonstração. Seja $S$ um subconjunto maximal de $V(G)$, com a propriedade de ser uma solução de $T(G ; X)=0$. Suponha, por um momento, que $G \backslash S$ possua uma componente par $C$. Seja v um vértice de $C$ cuja remoção de $C$ não defina mais que uma componente conexa a partir de $C$.

Como $\omega(G \backslash\{S \cup\{v\}\})=\omega(G \backslash S)+1$ e $S$ é uma solução de $T(G ; X)=0$, temos que $\{S \cup\{v\}\}$ também é uma solução, porque

$$
\begin{aligned}
0 & =|V(G)|+|S|-\omega(G \backslash S)-2 v(G) \\
& =|V(G)|+|S|-(\omega(G \backslash\{S \cup\{\mathrm{v}\}\})-1)-2 v(G) \\
& =|V(G)|+|S|+1-\omega(G \backslash\{S \cup\{\mathrm{v}\}\})-2 v(G) \\
& =|V(G)|+|S \cup\{v\}|-\omega(G \backslash\{S \cup\{\mathrm{v}\}\})-2 v(G),
\end{aligned}
$$

que contraria a maximalidade de $S$. 
Por exemplo, seja $G$ um grafo com $V(G)=\{a, b, c, x, y\}$, onde $d_{G}(a)=d_{G}(b)=d_{G}(c)=1, d_{G}(x)=2$ e $d_{G}(y)=3$. Os conjuntos $S=\{$ y $\}$ e $T=\{x, y\}$ são soluções para $T(G ; X)=0$. O grafo $G \backslash S$ tem componentes tanto pares como ímpares, mas o grafo $G \backslash T$ tem apenas componentes ímpares, pois, $T$ é maximal.

Proposiçãa 2.2. [Die97, p.41, ex.10] Seja $G=(V(G), E(G))$ um grafo. Então, $G$ contém um k-emparelhamento se, e somente se, $\omega(G \backslash X) \leq|X|+|V(G)|-2 k, \forall \mathrm{X} \subseteq V(G)$.

\section{Demonstração.}

A condição necessária é imediata.

Para provar a condição suficiente, admitamos que para todos os subconjuntos $S \subseteq V(G)$ se verifica $\omega(\mathrm{G} \backslash S) \leq|S|+t$, onde, $t=|V(G)|-2 k$.

Seja $H=G \bullet K_{t}$ o grafo com $V(H)=V(G) \cup V\left(K_{t}\right)$ e $E(H)=E(G) \cup E\left(K_{t}\right) \cup\{u v: \mathrm{u} \in \mathrm{V}(\mathrm{G})$ e $v \in V\left(K_{t}\right)$, resultando $G$ um subgrafo de $H$.

Em $G$ haverá um emparelhamento conectando todos os vértices menos os $t$, se e somente se, $H$ possui um emparelhamento perfeito.

Pelo teorema 1.1, $H$ tem emparelhamento perfeito se, e somente se, $\omega(H \backslash T) \leq|T|$ para todo $T \subseteq V(H)$.

Provemos que $\omega(H \backslash T) \leq|T|, \forall T \subseteq V(H)$

Consideremos dois casos:

$$
\begin{aligned}
& \text { (i) } V\left(K_{t}\right) \subset T \\
& \text { (ii) } V\left(K_{t}\right) \not \subset T \text {. }
\end{aligned}
$$

(i) Para $S \subset V(G)$ seja $T=S \cup V\left(K_{t}\right)$, temos $\omega(H \backslash T)=\omega(G \backslash S) \leq|S|+t=|T|$.

(ii) $\mathrm{O}$ grafo $H \backslash T$ é conexo, então $\omega(H \backslash T) \leq 1$ o que implica em $\omega(H \backslash T) \leq|T|$.

Portanto, $H$ tem um emparelhamento perfeito.

$\forall X \subseteq V(G)$. Pelo teorema 1.2, $2 k \leq 2 v(G)=\min _{X \subseteq V(G)}|V(G)|+|X|-\omega(G \backslash X)$.

Concluímos assim que $G$ possui um k-emparelhamento e a proposição está demonstrada.

Sabemos que as expressões $|V(G)|+|X|-\omega(G \backslash X)$ e $2 v(G)$ são inteiros pares, além disso, para todo subconjunto maximal $X \subseteq V(G)$, o grafo $G \backslash X$ não tem componentes pares. Porém, formalizamos a seguinte questão: Sob que condição um dado grafo possui pelo menos um subconjunto $X$ não vazio de vértices, tal que $X$, seja a solução da equação de Tutte-Berge? A seção 3 está constituída pela resposta, ou seja a caracterização dos grafos imersiveis, com este propósito estendemos o conceito de conjunto antifator de Summer [Sum76], que também é mencionado como o conjunto de Tutte por Lovász \& Plummer [Lov86]. 


\section{A Caracterização de Grafos Imersíveis}

Nesta seção, provamos o Teorema Principal, que fornece uma caracterização dos grafos imersiveis pelos conjuntos antifatores completos. Primeiramente, introduzimos a noção do conjunto antifator completo e logo em seguida, provamos a seguinte afirmação: todo subconjunto de vértices de um grafo $G$ que verifica a condição estrita de Tutte, não é uma solução para a equação associada de $G$.

Definição 3.1. Dado o grafo $G=(V(G), E(G)), S \subseteq V(G)$ é chamado um antifator completo de $G$ (ou conjunto completo de Tutte) se $\omega(G \backslash S) \geq|S|$.

Proposição 3.1. Para qualquer membro $G \operatorname{de~}^{\mathrm{n}}(m, v)$ seja $S$ um conjunto de vértices de $G$. Se $S$ satisfaz a desigualdade estrita da condição de Tutte, isto é, $\omega(G \backslash S)<|S|$, então $S$ não é uma solução da equação de Tutte-Berge $T(G ; X)=0$ associada de $G$.

\section{Demonstração.}

Suponhamos que $S$ seja uma solução de $T(G ; X)=0$ satisfazendo a condição estrita de Tutte.

De $\omega(G \backslash S)<|S|$ temos que $|V(G)|<|V(G)|+|S|-\omega(G \backslash S)$. [1]

Como $S$ é uma solução de $T(G ; X)=0$, então $|V(G)|+|S|-\omega(G \backslash S)=2 v(G)$.

De [1] e [2], obtemos $|V(G)|<2 v(G)$, o que contradiz o fato de que $2 v(G) \leq|V(G)|$ e está concluída a demonstração da proposição.

Teorema 3.1. Seja $G$ um membro de $\mathrm{H}^{\mathrm{n}}(m, v)$. Então, o grafo $\mathrm{G}$ é imersivel se, e somente se, $G$ possui antifator completo não vazio.

\section{Demonstração.}

Condição necessária, assumamos que $G$ seja imersivel e provemos que $G$ possui antifator completo.

Pela hipótese existe um subconjunto não vazio $S$ de $V(G)$, tal que, no corolário 1.1 seu máximo é atingido em $S$. Como o máximo é sempre não negativo ou seja, pelo menos é zero, então $|S| \leq \omega(G \backslash S)$.

Portanto, $S$ é um antifator completo para $G$.

Para a prova de suficiência, admitamos que $G$ possui antifator completo e provemos que este conjunto é uma solução da equação de Tutte-Berge $T(G ; X)=0$ associada de $G$.

Suponhamos que $G$ seja não imersivel e admitamos que existe um subconjunto não vazio $S \subset V(G)$ tal que $S$ verifica $\omega(G \backslash S) \geq|\mathrm{S}|$ de onde escrevemos, $0 \leq \omega(G \backslash S)-|S|$.

A equação de Tutte-Berge $T(G ; X)=0$ associada de $G$ possui a única solução $\varnothing$, isto é,

$$
|V(G)|-2 v(G)=\omega(G \backslash \varnothing)-|\varnothing|=\max _{X \subseteq V(G)}\{\omega(G \backslash X)-|X|\} .
$$

Por conseguinte temos que, $0 \leq \omega(G \backslash S)-|S|<\omega(G \backslash \varnothing)-|\varnothing|=\omega(G)$.

Sendo $G$ conexo, as desigualdades acima obrigam que $\omega(G)$ seja igual a um, de onde concluímos que $|V(G)|-2 v(G)=1$ e, portanto, que $|V(G)|$ é ímpar. Por outro lado, isto 
implica que $|S|-\omega(G \backslash S)$ seja nulo e, conseqüentemente, par. Mas, desta forma, o número inteiro $|V(G)|+|S|-\omega(G \backslash S)$ será ímpar, contrariando a proposição 2.1. Isto se deve à suposição de que $G$ não é imersivel.

Por conseguinte, $G$ é imersivel e está completada a demonstração do teorema.

Corolário 3.1. Sejam os membros $\mathrm{K}_{\mathrm{n}}, \mathrm{K}_{1, \mathrm{n}}$ e $\mathrm{C}_{\mathrm{n}}$ de $\mathrm{H}^{\mathrm{n}}(m, v(G))$, então eles são imersiveis, para todo inteiro par positivo $n$.

\section{Demonstração.}

Para qualquer vértice $x$ de $\mathrm{K}_{\mathrm{n}}\left(\mathrm{C}_{\mathrm{n}}\right)$ conjunto unitário com o único elemento $x$, é um conjunto antifator completo. Analogamente, o conjunto unitário gerado pelo elemento centro de $\mathrm{K}_{1, \mathrm{n}}$ é um conjunto antifator completo de $\mathrm{K}_{1, \mathrm{n}}$.

Seja $G=(V(G), E(G))$ um grafo. Para $\mathrm{v}_{\mathrm{o}}, \mathrm{v}_{\mathrm{t}} \in V(G)$, um $\left(\mathrm{v}_{\mathrm{o}}, \mathrm{v}_{\mathrm{t}}\right)$-caminho $P$ de $G$ é uma seqüência alternada de vértices e arestas

$$
\mathrm{v}_{\mathrm{o}}, \mathrm{v}_{\mathrm{o}} \mathrm{v}_{1}, \mathrm{v}_{1}, \mathrm{v}_{1} \mathrm{v}_{2}, \mathrm{v}_{2}, \ldots, \mathrm{v}_{\mathrm{t}-1}, \mathrm{v}_{\mathrm{t}-1} \mathrm{v}_{\mathrm{t}}, \mathrm{v}_{\mathrm{t}},
$$

onde, $V(P)=\left\{\mathrm{v}_{\mathrm{i}} \in V(G) / 0 \leq i \leq t\right\}, \mathrm{v}_{\mathrm{i}} \neq \mathrm{v}_{\mathrm{j}}$ para $i \neq j$, e $E(P)=\left\{\mathrm{v}_{\mathrm{i}} \mathrm{v}_{\mathrm{i}+1} \in E(G) / 0 \leq \mathrm{i} \leq \mathrm{t}-1\right\}$. Os vértices $\mathrm{v}_{\mathrm{o}}$ e $\mathrm{v}_{\mathrm{t}}$ são os extremos de $P$.

Uma árvore é um grafo conexo e acíclico, e uma folha (ou vértice pendente) de uma árvore é um vértice de grau um.

Corolário 3.2. Consideremos que $G$ é um membro de $\mathrm{H}^{\mathrm{n}}(m, v(G))$. Se $G$ é uma árvore, então $G$ é imersivel para todo inteiro positivo $n$.

\section{Demonstração.}

Toda árvore com pelo menos dois vértices possui pelo menos duas folhas. Seja v uma folha de uma árvore $G$ com $n$ vértices, então o grafo $G \backslash\{\mathrm{v}\}$, é também uma árvore com $n-1$ vértices. Pois, um vértice de grau um não pertence ao caminho que conecta outros dois vértices. Por conseguinte, para $x, y \in V(G \backslash\{\mathrm{v}\})$, cada $(x, y)$-caminho de $G$ é também um caminho de $G \backslash\{\mathrm{v}\}$. Logo, $G \backslash\{\mathrm{v}\}$ é conexo. Como uma remoção de um vértice não pode criar um ciclo, $G \backslash\{\mathrm{v}\}$ também é acíclico. Assim, $G \backslash\{\mathrm{v}\}$ é uma árvore com $n-1$ vértices.

Tomando-se o conjunto $S=\{x\}$, onde $x$ é uma folha de $G$, e $S$ verifica a equação de TutteBerge associado de $G$, está provado o corolário.

\section{Grafos Fatoráveis Constituem uma Subclasse dos Imersíveis}

Nesta última seção, apresentamos o teorema seguinte que prova que os grafos fatoráveis formam uma subclasse dos grafos imersíveis.

Teorema 4.1. Seja $G$ um membro de $\mathrm{H}^{\mathrm{n}}(m, v(G))$. Se $G$ possui emparelhamento perfeito, então $G$ é imersível. 


\section{Demonstração.}

Seja $G=(V(G), E(G))$ um grafo com um emparelhamento perfeito, então o número de vértices de $G$ é par.

Como por hipótese $G$ é conexo, sempre existe em $G$ um vértice u que, ao ser removido de $G$ produz outro grafo G-u que é conexo. Logo, para $X=\{u\}$, a equação de Tutte-Berge reduz-se a:

$$
|V(G)|+|X|-\omega(G \backslash X)-2 v(G)=|V(G)|+1-1-2 \frac{|V(G)|}{2}=0
$$

Portanto, $G$ é imersivel e o teorema está demonstrado.

\section{Referências Bibliográficas}

[Ber57] Berge, C. (1957). Sur le couplage maximum d'un graphe. Proc. Nat. Science, 247, p.258.

[Boa03] Boaventura Netto, P.O. (2003). GRAFOS: Teoria, Modelos e Algoritmos. $3^{\mathrm{a}}$ ed., Edgard Blúcher, São Paulo.

[Bon95] Bondy, J.A. (1995). Matchings and Extensions. In: Handbook of Combinatorics [edited by R.I. Graham, M. Grótschel and L. Lovász], vol. I, 179-232, North-Holland, Amsterdam.

[Bru87] Brualdi, R.A. (1987). Introduction to Matching Theory. In: Combinatorial Geometries (Encyclopedia of mathematics and its applications; v.29) [edited by N. White], 53-69, Cambridge University Press, Cambridge.

[Cor88] Cornuéjols, G. (1988). General Factors of Graphs. J. Comb. Theory Ser. (B), 45, 449-467.

[Cor99] Cortez-Morales, W.J. (1999). G-Comportamento dos Matchings, Matróides e Fluxos. Tese de Livre-Docente, Universidade Estadual Paulista, Campus de São José do Rio Preto - SP.

[Die97] Diestel, R. (1997). Graph Theory. Grad. Texts Math. 173, Springer-Verlag, New York, Inc.

[Lov86] Lovász, L. \& Plummer, M.D. (1986). Matching Theory. Annals of Discrete Mathematics, 29.

[Sum76] Summer, D.P. (1976). 1-factors and antifactor sets. J. London Math. Soc., 2(3), 351-359.

[Tut47] Tutte, W.T. (1947). The factorization of linear graphs. J. London Math. Soc., 22, 107-111. 